\title{
Fatores Associados à Densidade Mamográfica de Mulheres na Pós-Menopausa
}

\author{
Factors Associated with Mammographic Density in Postmenopausal Women \\ Renata Ferreira Cleto Bittencourt Siqueira, Danielle Santos Bezerra Sá, \\ Aarão Mendes Pinto Neto, César Cabello, Délio Marques Conde \\ Lúcia Helena Simões da Costa Paiva, Gislaine Aparecida Fonsechi-Carvasan
}

\begin{abstract}
RESUM0
Objetivo: avaliar a associação de fatores epidemiológicos, antropométricos, reprodutivos $e$ hormonais com a densidade mamográfica de mulheres na pós-menopausa.

Métodos: estudo retrospectivo, tipo corte transversal, incluindo 144 mulheres com idade igual ou superior a 45 anos, com no mínimo 12 meses de amenorréia e não usuárias de terapia de reposição hormonal nos últimos seis meses. Foram revisados os prontuários médicos, avaliando-se: idade, peso, altura, índice de massa corpórea (IMC), paridade, idade à menarca, idade à menopausa e os niveis dos hormônios estradiol, folículo-estimulante (FSH) e luteinizante. As mamografias foram analisadas nas incidências crânio-caudal e médio-lateral obliqua por dois examinadores, de forma cega, sendo classificadas em densas e não densas, segundo os critérios de Wolfe. Na análise estatística, utilizou-se a freqüência, mediana, valores mínimo e máximo, teste de Wilcoxon e odds ratio. Foi realizada análise de regressão logística múltipla, utilizando o processo de seleção passo a passo, com nível de significância de 5\%.

Resultados: a freqüência de mamas densas foi de $45 \%$. As mulheres com mamas consideradas densas apresentaram menor peso $(60,5 \mathrm{vs} 71,9 \mathrm{~kg}-p<0,01)$, menor IMC $\left(25,9 \mathrm{vs} 31,0 \mathrm{~kg} / \mathrm{m}^{2}\right.$ - p<0,01), menor tempo de pós-menopausa $(6,0$ vs 10,0 anos - $p<0,01)$ e maiores níveis de FSH (75,2 vs $60,3 \mathrm{mU} / \mathrm{mL}-p<0,01)$. A probabilidade de apresentar mamas densas diminuiu em mulheres com peso superior a $67 \mathrm{~kg}(O R=4,0$; IC 95\%: 1,50-10,66), IMC superior a 30 $\mathrm{kg} / \mathrm{m}^{2}(\mathrm{OR}=6,69$; IC 95\%: 2,54-17,65), com mais de dois partos $(\mathrm{OR}=7,84$; IC 95\%: 1,6736,81), tempo de pós-menopausa maior ou igual a sete anos (OR = 2,05; IC 95\%: 1,05-3,99) e niveis de FSH inferiores a 134,8 $\mathrm{mU} / \mathrm{mL}$.

Conclusão: o peso, IMC, paridade, o tempo de menopausa e níveis de FSH associaram-se significativamente aos padrões de densidade mamográfica.
\end{abstract}

PALAVRAS-CHAVE: Densidade mamária. Menopausa. Índice de massa corpórea. Mamografia. Mama: câncer.

\section{Introdução}

A mama é composta por quantidade variável de tecidos adiposo, conjuntivo e epitelial, que

Departamento de Tocoginecologia, Faculdade de Ciências Médicas - Universidade Estadual de Campinas

Correspondência:

Aarão Mendes Pinto Neto

Departamento de Tocoginecologia/CAISM/UNICAMP

Rua Alexander Fleming, 101 - Cidade Universitária "Zeferino

Vaz"

13083-970 - Campinas - SP

Tel: (19) 3788-9354 - FAX: (19) 3788-9354

e-mail: aarão@obelix.unicamp.br vão compor a sua densidade radiológica ${ }^{1,2}$. Como resultado da composição histológica da mama, observam-se duas densidades na mamografia: densidade de gordura, que reflete a quantidade de tecido adiposo e densidade de água, que reflete o conteúdo de tecidos conjuntivo e glandular da mama. A densidade de gordura é evidenciada à mamografia pela hipertransparência e radiolucência, enquanto a densidade de água é evidenciada pela hipotransparência ${ }^{1}$. A formação da imagem radiológica da mama resulta da superposição destes tecidos, visualizados à mamografia pelas diferentes densidades. Quanto maior o conteúdo de 
gordura da mama, menor a densidade mamográfica, ao passo que a maior proporção de tecidos conjuntivo e epitelial aumenta a densidade radiológica da mama ${ }^{3}$.

Baseado na proporção dos tecidos que compõem a mama, Wolfe ${ }^{4}$ classificou os padrões mamográficos em quatro categorias: N1, P1, P2 e DY. Os padrões P2 e DY são os que apresentam maior densidade mamográfica e são considerados de alto risco para o desenvolvimento de câncer de mama ${ }^{5,6}$. A densidade mamária sofre modificações em função da idade, estado menopausal, paridade, altura, peso corpóreo e uso de terapia de reposição hormonal (TRH). Possiveis associações com o estado nutricional, prática de exercícios, alcoolismo e história familiar de câncer de mama foram relatadas ${ }^{7}$. Stomper et al. ${ }^{8}$ observaram mudanças na densidade mamária em função da idade, havendo substituição gradual do tecido fibroglandular por tecido adiposo. Estes autores verificaram que mulheres com menos de duas gestações apresentavam tendência a ter mamas densas. A importância do estudo da densidade mamária baseia-se em dois aspectos: no aumento do risco para câncer de mama e na diminuição da sensibilidade e especificidade da mamografia $^{9,10}$

As evidências de que as características mamográficas do parênquima mamário forneçam informações sobre o risco de desenvolvimento de câncer de mama aumentaram nos dias atuais. Estudo recente no qual se avaliou a influência da TRH estroprogestativa na incidência do câncer de mama e sobre as mamografias de mulheres na pós-menopausa verificou que os casos de câncer de mama em usuárias de TRH foram diagnosticados em estádios mais avançados, quando comparados com os do grupo placebo. A hipótese dos autores deste estudo foi que a TRH, além de estimular o crescimento tumoral, esteve associada ao atraso no diagnóstico, possivelmente por dificuldades na interpretação das mamografias, decorrentes do aumento na densidade mamária ${ }^{11}$. Estudos prévios também observaram a associação entre o aumento da densidade mamária e o atraso na detecção do câncer de mama ${ }^{6,10,12}$.

A mamografia tem sido recomendada às mulheres que procuram atendimento especializado por questões relacionadas à menopausa, não só pela idade, mas também por serem potenciais candidatas ao uso da TRH. Considerando-se as modificações que podem ocorrer na densidade mamográfica em função da idade, peso, paridade, estado menopausal e do uso da TRH, torna-se importante conhecer os fatores associados à densidade radiológica da mama, buscando-se medidas que possam minimizar as conseqüências dessas modificações.

O objetivo desse estudo foi avaliar a associação de fatores epidemiológicos, antropométricos, reprodutivos e hormonais com a densidade mamográfica de mulheres na pós-menopausa. Apesar de existirem estudos na literatura sobre o tema, esse, até onde sabemos, é o primeiro estudo nacional que avalia tal associação com fatores hormonais.

\section{Pacientes e Métodos}

Realizou-se estudo retrospectivo, tipo corte transversal, revisando-se os prontuários médicos e as mamografias de mulheres atendidas no Ambulatório de Menopausa do Centro de Atenção Integral à Saúde da Mulher (CAISM) da Universidade Estadual de Campinas (Unicamp). Os critérios de inclusão utilizados foram: mulheres com idade de 45 anos ou mais, com tempo mínimo de 12 meses de amenorréia e não usuárias de TRH nos últimos seis meses. Foram excluídas as mulheres com antecedente de cirurgia mamária, antecedente pessoal de câncer de mama, achado mamográfico de lesão suspeita ou aquelas que não apresentassem mamografia de boa qualidade. Na avaliação da qualidade da mamografia, consideraram-se o grau de definição e contraste da imagem e a presença ou não de artefatos que impedissem a avaliação da mamografia. As mamografias foram realizadas em diferentes serviços. Foram avaliados 144 prontuários com as respectivas mamografias realizadas quando da admissão no serviço.

Para o cálculo do tamanho amostral considerou-se a proporção de mulheres com mais de 50 anos e com mamas densas (P2/DY) de 40\% ${ }^{13}$. O tamanho amostral de 144 mulheres permitiu gerar intervalos de confiança de $95 \%(\alpha=5 \%)$ com semi-amplitude de $8 \%$.

A variável dependente considerada foi a densidade mamográfica do parênquima mamário classificada segundo Wolfe ${ }^{4}$ : N1 (menor radiopacidade), padrão predominantemente adiposo com mama composta quase inteiramente por gordura; P1, padrão fibroductoglandular com ductos proeminentes ocupando até um quarto do volume da mama; P2, padrão fibroductoglandular com ductos proeminentes ocupando mais que um quarto do volume da mama; DY (maior radiopacidade), padrão fibroglandular ocupando quase toda a mama. Estes padrões foram agrupados, e a densidade mamográfica foi categorizada como não densa (N1/ $\mathrm{P} 1)$ e densa (P2/DY). 
As variáveis independentes consideradas foram: idade, peso, altura, índice de massa corpórea (IMC), paridade, idade à menarca, idade à menopausa, tempo de menopausa e concentração plasmática de estradiol, hormônio luteinizante (LH) e hormônio folículo-estimulante (FSH). Para verificação dos fatores associados à densidade mamográfica, o peso foi categorizado de acordo com o quartil e a paridade em nulíparas, um parto e dois ou mais partos. O IMC foi expresso $\mathrm{em} \mathrm{kg} / \mathrm{m}^{2} \mathrm{e}$ categorizado em normal (18,5-24,9), sobrepeso $(25,0-29,9)$, obesidade classe I $(30,0-34,9)$ e obesidade classe II $(335,0){ }^{14}$. Os níveis hormonais foram considerados de acordo com as concentrações para a menopausa: FSH entre 25 e 134,8 mUI/ml, LH entre 7,7 e $58,5 \mathrm{mUI} / \mathrm{mL}$ e estradiol inferior a 30 $\mathrm{pg} / \mathrm{mL}$. As demais variáveis foram categorizadas de acordo com a mediana da população total do estudo (144 mulheres). As dosagens hormonais foram realizadas quando da admissão no serviço, pela técnica de eletroquimioluminescência.

As mamografias de ambas as mamas foram avaliadas nas incidências médio-lateral oblíqua e crânio-caudal, de forma cega, por dois examinadores. Foram então classificadas segundo os padrões de Wolfe ${ }^{4}$. Quando houve assimetria no padrão de densidade entre as mamas de uma mesma paciente, prevaleceu a classificação de maior densidade. Os laudos não concordantes entre os dois primeiros examinadores foram avaliados por um terceiro examinador, também de forma cega.

Inicialmente realizou-se análise descritiva dos dados com cálculos da freqüência, mediana, valores mínimo e máximo e, para verificação das diferenças das características das mulheres segundo o padrão de densidade mamográfica, utilizou-se o teste não paramétrico de Wilcoxon. A partir das variáveis independentes foi feita a análise de regressão logística univariada (odds ratio bruto com seus respectivos intervalos de confiança de 95\%). Esta análise consistiu em correlacionar separadamente cada uma das variáveis independentes com a variável dependente (mama densa ou não densa), para identificar o quanto cada variável independente estava associada à densidade mamográfica. Em uma segunda etapa, foi realizada a análise de regressão logística múltipla, a fim de identificar quais variáveis estariam, conjuntamente, associadas ao padrão mamográfico ${ }^{15}$. Para isto utilizou-se o processo de seleção stepwise, que consiste em, a cada passo, eleger uma variável independente com maior evidência de explanar a dependente, verificando-se, posteriormente, se com a sua inclusão no modelo aquelas previamente escolhidas continuavam sendo significantes.
Esse estudo foi aprovado pela Comissão de Pesquisa do Centro de Atenção Integral à Saúde da Mulher e Comitê de Ética em Pesquisa da Faculdade de Ciências Médicas da Unicamp.

\section{Resultados}

Das 144 mulheres avaliadas, 65 (45\%) apresentaram mamas com padrão mamográfico denso (Tabela 1). As mulheres com mamas densas apresentaram mediana de peso de $60,5 \mathrm{~kg}$ e as com mamas não densas de $71,9 \mathrm{~kg}(\mathrm{p}<0,01)$. As medianas do IMC de mulheres com mamas densas e não densas foram de $25,9 \mathrm{~kg} / \mathrm{m}^{2}$ e $31,0 \mathrm{~kg} / \mathrm{m}^{2}$ $(\mathrm{p}<0,01)$, respectivamente. Em relação ao tempo de menopausa, verificou-se que a mediana foi de 6,0 anos para as mulheres com mamas densas e de 10,0 anos para as com mamas não densas $(\mathrm{p}<0,01)$. As medianas da idade, altura, paridade, idade à menarca e idade à menopausa não apresentaram diferenças estatisticamente significativas (Tabela 2).

Tabela 1 - Distribuição percentual das mulheres de acordo com o padrão de densidade mamográfica, segundo os critérios de Wolfe ${ }^{4}$.

\begin{tabular}{lrr} 
Densidade mamográfica & $\mathbf{n}$ & $\%$ \\
\hline Mamas não densas (N1/P1) & 79 & 55 \\
Mamas densas (N2/DY) & 65 & 45 \\
Total & 144 & 100 \\
\hline
\end{tabular}

Em relação aos niveis hormonais, observouse em mulheres com mamas densas mediana de FSH de 75,2 mUI/mL e, em mulheres com mamas não densas, de 60,3 mUI/mL (p<0,01); as medianas de $\mathrm{LH}$ foram de $28,5 \mathrm{mUI} / \mathrm{mL}$ e $22,3 \mathrm{mUI} / \mathrm{mL}$ em mulheres com mamas densas e não densas $(\mathrm{p}<0,01)$, respectivamente. Não houve diferença estatisticamente significativa nos níveis séricos de estradiol (Tabela 3).

Nas mulheres cujo peso variou entre 66,6 kg e 80,3 kg, 30,4\% apresentaram padrão mamográfico denso e 18,5\% padrão não denso (OR: 4,00; IC 95\%: 1,50-10,66). Nas mulheres com peso igual ou superior a $80,4 \mathrm{~kg}$, verificaram-se mamas não densas em $31,6 \%$ e mamas densas em 16,9\% (OR: 4,54; IC 95\%: 1,69-12,25). Quanto ao IMC, observou-se que entre os valores de $30,0 \mathrm{~kg} / \mathrm{m}^{2}$ e 34,9 $\mathrm{kg} / \mathrm{m}^{2}, 38 \%$ das mulheres apresentaram mamas densas e 15,4\% mamas não densas (OR: 6,69; IC 95\%: 2,54-17,65). O cálculo do odds ratio ajustado por todas as variáveis mostrou diferença estatisticamente significativa com o IMC igual ou superior a $30 \mathrm{~kg} / \mathrm{m}^{2}$. Entre as mulheres com dois ou 
mais partos, observou-se que $97,5 \%$ apresentaram mamas não densas e $83,1 \%$ mamas densas (OR: 7,84; IC 95\%: 1,67-36,81). O tempo de menopausa associou-se significativamente ao padrão de densidade mamográfica, sendo que entre as mulheres com sete anos ou mais de menopausa, 60,8\% apresentaram mamas não densas e 43,1\% mamas densas (OR: 2,05; IC 95\%: 1,05-3,99). Dentre as mulheres com niveis séricos de FSH igual ou superior a 134,8 $\mathrm{mUI} / \mathrm{ml}, 2,5 \%$ apresentaram mamas não densas e 12,3\% mamas densas (OR: 0,19; IC 95\%: 0,04-0,91) (Tabela 4). O cálculo do odds ratio ajustado por todas as variáveis mostrou que o IMC e a paridade associaram-se de forma significativa com o padrão de densidade mamográfica (Tabelas 4 e 5).

Tabela 2 - Comparação das características epidemiológicas, antropométricas e reprodutivas entre mulheres com mamas densas e não densas $(\mathrm{n}=144)$.

\begin{tabular}{|c|c|c|c|c|c|}
\hline \multirow[t]{2}{*}{ Variáveis } & \multirow[t]{2}{*}{ Densidade mamográfica } & \multirow[t]{2}{*}{ Mediana } & \multicolumn{2}{|c|}{ Variação } & \multirow[t]{2}{*}{ Valor de $p$} \\
\hline & & & Mínimo & Máximo & \\
\hline \multirow{2}{*}{ Idade (anos) } & Densa & 54,0 & 45,0 & 68,0 & \multirow{2}{*}{0,08} \\
\hline & Não densa & 55,0 & 45,0 & 78,0 & \\
\hline \multirow{2}{*}{ Peso (kg) } & Densa & 60,5 & 40,3 & 105,5 & \multirow{2}{*}{$<0,01$} \\
\hline & Não densa & 71,9 & 49,0 & 131,0 & \\
\hline \multirow{2}{*}{ Altura (m) } & Densa & 1,5 & 1,4 & 1,8 & \multirow{2}{*}{0,68} \\
\hline & Não densa & 1,5 & 1,4 & 1,7 & \\
\hline \multirow{2}{*}{$\mathrm{IMC}\left(\mathrm{kg} / \mathrm{m}^{2}\right)^{\dagger}$} & Densa & 25,9 & 17,4 & 41,7 & \multirow{2}{*}{$<0,01$} \\
\hline & Não densa & 31,0 & 19,2 & 50,4 & \\
\hline \multirow{2}{*}{ Paridade } & Densa & 3,0 & 1,0 & 14,0 & \multirow{2}{*}{0,05} \\
\hline & Não densa & 4,0 & 1,0 & 13,0 & \\
\hline \multirow{2}{*}{ Idade à menarca (anos) } & Densa & 13,0 & 9,0 & 18,8 & \multirow{2}{*}{0,24} \\
\hline & Não densa & 13,0 & 9,0 & 18,8 & \\
\hline \multirow{2}{*}{ Idade à menopausa (anos) } & Densa & 48,0 & 30,0 & 57,0 & \multirow{2}{*}{0,43} \\
\hline & Não densa & 47,0 & 30,0 & 55,0 & \\
\hline \multirow{2}{*}{ Tempo de menopausa (anos) } & Densa & 6,0 & 1,0 & 28,0 & \multirow{2}{*}{$<0,01$} \\
\hline & Não densa & 10,0 & 1,0 & 27,0 & \\
\hline
\end{tabular}

${ }^{\dagger}$ IMC: índice de massa corpórea.

"Excluídas duas mulheres com número de partos desconhecido.

*Teste de Wilcoxon.

Tabela 3 - Comparação dos valores de estradiol $(\mathrm{pg} / \mathrm{mL})$, FSH $(\mathrm{mUl} / \mathrm{mL})$ e $\mathrm{LH}(\mathrm{mUl} / \mathrm{mL})$ entre mulheres com mamas densas e não densas $(\mathrm{n}=144)$

\begin{tabular}{|c|c|c|c|c|c|}
\hline \multirow[t]{2}{*}{ Variáveis } & \multirow[t]{2}{*}{ Densidade mamográfica } & \multirow[t]{2}{*}{ Mediana } & \multicolumn{2}{|c|}{ Variação } & \multirow[b]{2}{*}{ Valor de $p^{*}$} \\
\hline & & & Mínimo & Máximo & \\
\hline \multirow{2}{*}{ Estradiol $(p g / m L)^{\#}$} & Densa & 19,9 & 5,0 & 205,7 & \multirow[t]{2}{*}{0,51} \\
\hline & Não densa & 19,9 & 4,9 & 158,0 & \\
\hline \multirow{2}{*}{ FSH (mUl/mL) } & Densa & 75,2 & 16,6 & 200,0 & \multirow{2}{*}{$<0,01$} \\
\hline & Não densa & 60,3 & 3,2 & 193,0 & \\
\hline \multirow{2}{*}{ LH (mUl/mL) } & Densa & 28,5 & 3,8 & 98,6 & \multirow{2}{*}{$<0,01$} \\
\hline & Não densa & 22,3 & 0,1 & 68,0 & \\
\hline
\end{tabular}

"Excluídas duas mulheres com concentração de estradiol ignorada.

'Teste de Wilcoxon. 
Tabela 4 - Fatores associados à densidade mamográfica. Análise bivariada $(n=144)$

\begin{tabular}{|c|c|c|c|c|c|}
\hline \multirow[t]{3}{*}{ Fatores } & \multicolumn{4}{|c|}{ Densidade Mamográfica } & \multirow[t]{3}{*}{ odds ratio (IC 95\%) } \\
\hline & \multicolumn{2}{|c|}{ Não densa } & \multicolumn{2}{|c|}{ Densa } & \\
\hline & n (79) & $\%$ & n (65) & $\%$ & \\
\hline \multicolumn{6}{|l|}{ Peso (kg) } \\
\hline$\leq 56,7$ & 12 & 15,2 & 24 & 36,9 & Ref. \\
\hline 56,8 a 66,5 & 18 & 22,8 & 18 & 27,7 & $2,00(0,77-5,18)$ \\
\hline 66,6 a 80,3 & 24 & 30,4 & 12 & 18,5 & $4,00(1,50-10,66)$ \\
\hline$\geq 80,4$ & 25 & 31,6 & 11 & 16,9 & $4,54(1,69-12,25)$ \\
\hline \multicolumn{6}{|c|}{ Tempo de menopausa (anos) } \\
\hline$\leq 6$ & 31 & 39,2 & 37 & 56,9 & Ref. \\
\hline$\geq 7$ & 48 & 60,8 & 28 & 43,1 & $2,05(1,05-3,99)$ \\
\hline \multicolumn{6}{|l|}{ FSH (mUl/mL) } \\
\hline$\leq 134,7$ & 77 & 97,5 & 57 & 87,7 & Ref. \\
\hline$\geq 134,8$ & 2 & 2,5 & 8 & 12,3 & $0,19(0,04-0,91)$ \\
\hline
\end{tabular}

Tabela 5 - Fatores significativamente associados à densidade mamográfica. Regressão logística $(n=144)$.

\begin{tabular}{|c|c|c|c|c|c|c|}
\hline \multirow[t]{3}{*}{ Fatores } & \multicolumn{4}{|c|}{ Densidade Mamográfica } & \multirow[t]{3}{*}{ odds ratio (IC 95\%) } & \multirow[t]{3}{*}{ odds ratio ajustado* (IC 95\%) } \\
\hline & \multicolumn{2}{|c|}{ Não densa } & \multicolumn{2}{|c|}{ Densa } & & \\
\hline & n (79) & $\%$ & $n(65)$ & $\%$ & & \\
\hline \multicolumn{7}{|l|}{ IMC $\left(\mathrm{kg} / \mathrm{m}^{2}\right)^{\#}$} \\
\hline$\leq 24,9$ & 13 & 16,5 & 29 & 44,6 & Ref. & Ref. \\
\hline 25 a 29,9 & 18 & 22,8 & 21 & 32,3 & $1,91(0,77-4,74)$ & $1,94(0,76-4,91)$ \\
\hline 30 a 34,9 & 30 & 38,0 & 10 & 15,4 & $6,69(2,54-17,65)$ & $5,96(2,23-15,96)$ \\
\hline$\geq 35$ & 18 & 22,8 & 5 & 7,7 & $8,03(2,45-26,33)$ & $7,51(2,24-25,21)$ \\
\hline \multicolumn{7}{|l|}{ Paridade } \\
\hline 0 & 0 & 0,0 & 2 & 3,1 & \multirow{2}{*}{ Ref. } & \multirow{2}{*}{ Ref. } \\
\hline 1 & 2 & 2,5 & 9 & 13,8 & & \\
\hline$\geq 2$ & 77 & 97,5 & 54 & 83,1 & $7,84(1,67-36,81)$ & $5,87(1,17-28,58)$ \\
\hline
\end{tabular}

\#IMC: Índice de massa corpórea.

*Regressão logística através da seleção stepwise.

\section{Discussão}

O objetivo desse estudo foi avaliar os fatores clínicos e hormonais associados à densidade mamográfica de mulheres na pós-menopausa. Verificou-se que número significativo de mulheres no início do acompanhamento médico apresentava padrão mamográfico denso. Deve-se estar atento para este resultado, uma vez que mulheres com maior densidade mamária não só apresentam um aumento no risco para câncer de mama ${ }^{9}$, mas também podem apresentar atraso no diagnóstico mamográfico do câncer ${ }^{9}$.
A freqüência de mamas densas observada na presente casuística foi de $45 \%$, maior que a relatada para esta faixa etária por Bergkvist et al. ${ }^{16}$. Porém, os resultados nesse estudo foram observados em população que procurou atendimento especializado por questões relativas à menopausa, ao passo que Bergkvist et al. ${ }^{16}$ realizaram estudo populacional com mulheres suecas com mais de 40 anos convidadas a participar de um programa de rastreamento mamográfico, no qual também foram avaliados os fatores epidemiológicos associados ao padrão mamográfico denso.

A idade nas faixas estudadas não está associada com os padrões de densidade mamográfica. 
Cabe ressaltar, no entanto, que a variável tempo de menopausa (colinear com a idade cronológica) associou-se ao padrão de densidade mamográfica. Nas mulheres com mamas densas, a mediana do tempo de menopausa foi significativamente menor que o das com mamas não densas. Mamografias com padrão denso são comumente observadas em mulheres jovens, mas sua freqüência diminui com o aumento da idade cronológica, especialmente após a menopausa ${ }^{17}$.

Bergkvist et al. ${ }^{16}$ observaram que $65 \%$ das mulheres de 35 anos apresentam mamas com padrão denso (definido como mais de 25\% da área mamária contendo parênquima denso), ao passo que a proporção de mulheres por volta de 50 anos com padrão denso à mamografia é de aproximadamente 35\%, e em mulheres em torno dos 55 anos é de 15 a $20 \%$. Considerando que o tempo de pós-menopausa é colinear com a idade cronológica da mulher, não se deve concluir que a idade não influenciou o padrão de densidade do parênquima mamário. A associação do tempo de pós-menopausa com os padrões de densidade mamográfica foi confirmada quando se calculou o odds ratio bruto, porém não persistiu quando o odds ratio foi ajustado por todas variáveis, ou seja, apesar de ser um importante fator, não foi influenciado por outras variáveis.

Uma explicação para o fato de o padrão mamográfico denso associar-se a maior tempo de menopausa, relaciona-se à diminuição dos níveis circulantes dos esteróides sexuais, especialmente dos estrogênios, que se verifica no período do climatério. Na presente casuística, apesar de os niveis plasmáticos de estradiol não terem apresentado associação com o padrão mamográfico, observamos que as mulheres com mamas densas apresentaram concentrações plasmáticas significativamente maiores dos hormônios folículo-estimulante e luteinizante, quando comparadas às mulheres com mamas não densas. Até onde sabemos, este foi o primeiro estudo nacional que verificou esta associação.

Os fatores antropométricos são amplamente relacionados com o padrão mamográfico: mulheres com maior peso corpóreo geralmente apresentam mamas não densas ${ }^{18}$. Ao contrário, mulheres com menor peso corpóreo freqüentemente apresentam mamas com padrão denso ${ }^{19}$. Nesse estudo, a probabilidade de uma mulher apresentar mamas densas variou de acordo com o peso corpóreo, sendo menor naquelas com peso superior a $66,6 \mathrm{~kg}$. O IMC associou-se ao padrão de densidade mamográfica. Valores de IMC inferiores a 30 $\mathrm{kg} / \mathrm{m}^{2}$ aumentaram a probabilidade de a mulher apresentar padrão mamográfico denso (sete a oito vezes). Esse resultado está de acordo com a literatura, que é quase unânime quanto a este aspecto ${ }^{2,19,20}$. Estes estudos apontam a altura e o peso como elementos preditivos da densidade mamográfica $^{20}$. Não houve diferenças significativas nas medianas da paridade de mulheres com mamas densas e não densas, porém quando se estratificou a paridade, confirmou-se que a multiparidade (dois filhos ou mais) estava associada a uma menor probabilidade de a mulher apresentar mamas densas. Este resultado foi concordante com os relatos de outros autores ${ }^{18,21}$.

Entre as limitações desse estudo, uma das principais foi o caráter retrospectivo da observação, o que não permitiu a inclusão da idade ao primeiro parto, o tempo de amamentação e a distribuição da gordura corporal, que são fatores sabidamente associados ao padrão de densidade mamográfica. Nem todas as mamografias foram realizadas no mesmo serviço, dificultando o controle de qualidade adequado das mesmas. Isso pode ter sido minimizado, quando se adotaram critérios para a avaliação da qualidade das mamografias. Porém, acreditamos que esse estudo poderá trazer alguma contribuição ao mostrar a alta freqüência de mamas densas já à primeira consulta, em serviço que atende mulheres na pós-menopausa e, portanto, candidatas ao uso de TRH. Embora não tenha sido o objetivo do presente estudo, a TRH pode estar associada ao aumento do risco de câncer de mama; soma-se a isto o fato de as mulheres com mamas densas poderem apresentar atraso no diagnóstico do câncer de mama, relacionado à diminuição da sensibilidade e especificidade da mamografia $^{21}$.

Apesar de não haver programas de rastreamento para câncer de mama no Brasil, pode-se observar que mulheres na pós-menopausa submetidas a mamografias são, geralmente, freqüentadoras de consultórios ou serviços públicos especializados no atendimento de mulheres climatéricas. Portanto, deve-se chamar a atenção dos profissionais que as assistem para os fatores associados ao padrão de densidade mamográfica. O conhecimento desses fatores poderá contribuir para a melhoria da qualidade da assistência.

Nesse sentido, o que pode ser feito para minimizar os efeitos do padrão mamográfico denso? Segundo Van Gils ${ }^{9}$, muito pouco. Recomendase atenção na realização e interpretação da mamografia, o uso de métodos complementares (ultra-sonografia, ressonância magnética), principalmente se houver achado suspeito durante o exame clínico das mamas, mas não visualizado na mamografia ${ }^{22}$. A mamografia deve ser interpretada por profissionais qualificados e com experiência em imaginologia mamária.

Orienta-se atenção quanto à técnica na realização da mamografia. Os serviços especializados em mamografia devem ter a qualidade do exame monitorada durante toda a seqüência de produção 
da imagem, incluindo o mamógrafo, processadora dedicada e a câmara escura. O posicionamento adequado é outro fator que merece atenção, uma vez que deve incluir a maior quantidade possivel de tecido mamário, diminuindo a possibilidade de exame falso-negativo. Outra recomendação refere-se à suspensão da TRH por período de no mínimo 3 semanas $^{23}$, previamente à realização da mamografia. Quando possivel, a utilização de outras modalidades terapêuticas ${ }^{24}$, que não a TRH, para a prevenção e tratamento de doenças associadas ao climatério, deve ser considerada. Não existe, até o momento, consenso da necessidade de se diminuir o intervalo de rastreamento do câncer de mama em mulheres com mamas densas.

Finalmente, consideramos que são necessários estudos prospectivos nacionais que avaliem a associação de cada fator estudado com a densidade do parênquima mamário ao longo do tempo. A associação entre padrão mamográfico denso e câncer de mama, bem como o atraso no diagnóstico do câncer de mama em mulheres com mamas densas, também deverão ser explorados em estudos prospectivos.

\section{ABSTRACT}

Purpose: to evaluate the relationship between epidemiologic, anthropometric, reproductive and hormonal factors and mammographic density in postmenopausal women.

Methods: this is a retrospective, cross-sectional study, including 144 women aged 45 years or more, with at least 12 months of amenorrhea and who were non users of hormone replacement therapy during the last six months. Medical charts were reviewed to evaluate age, weight, body mass index (BMI), parity, age at menarche, age at menopause and levels of estradiol, follicle stimulating (FSH) and luteinizing hormones. Mammograms were analyzed by two blinded investigators. The films were taken in the craniocaudal and mediolateral views and mammography was classified as dense and nondense, according to the Wolfe criteria. For statistical analysis, the frequency, median, minimum and maximum values, the Wilcoxon test and the odds ratio were used. Multiple logistic regression was performed, using the stepwise selection, with a 5\% significance level.

Results: the frequency of dense breasts was $45 \%$. Women with dense breasts were of lower weight $(60.5 \mathrm{vs} .71 .9 \mathrm{~kg}-p<0.01)$, had a lower BMI (25.9 vs $\left.31.0 \mathrm{~kg} / \mathrm{m}^{2}-p<0.01\right)$, a shorter time since onset of menopause $(6.0 \mathrm{vs} 10$ years $-p<0.01)$ and higher levels of FSH (75.2 vs $60.3 \mathrm{mU} / \mathrm{mL}-p<0.01)$. The probability of having dense breasts decreased in women whose weight was $67 \mathrm{~kg}$ or more $(O R=4.0, C I 95 \%=1.50$ 10.66), BMI was higher than $30 \mathrm{~kg} / \mathrm{m}^{2}(O R=6.69, \mathrm{CI} 95 \%=$ 1.67-36.81), time since onset of menopause was superior or equal to seven years $(O R=2.05, C I 95 \%=1.05-3.99)$ and FSH levels were lower than $134.8 \mathrm{mU} / \mathrm{mL}$.
Conclusion: weight, BMI, parity, time since menopause and FSH levels were significantly associated with mammographic density patterns.

KEYWORDS: Mammographic density. Menopause. Body mass index. Mammography. Breast cancer.

\section{Referências}

1. Byrne C, Schairer C, Wolfe J, et al. Mammographic features and breast cancer risk: effects with time, age, and menopause status. J Natl Cancer Inst 1995; 87:1622-9.

2. Sala E, Warren R, McCann J, Duffy S, Luben R, Day N. High-risk mammographic parenchymal patterns and anthropometric measures: a case control study. Br J Cancer 1999; 81:1257-61.

3. Bright RA, Morrisson AS, Brisson $\mathrm{J}$, et al. Relationship between mammographic and histologic features of breast tissue in women with benign biopsies. Cancer 1988; 61:266-71.

4. Wolfe JN. Breast patterns as an index of risk for developing breast cancer. Am J Roentgenol 1976; 126:1130-7.

5. Oza AM, Boyd NF. Mammographic parenchymal patterns: a marker of breast cancer risk. Epidemiol Rev 1993; 15:196-208.

6. Sala E, Warren R, McCann J, Duffy S, Day N, Luben R. Mammographic parenchymal patterns and mode of detection: implications for the breast screening programme. J Med Screen 1998; 5:207-12.

7. Boyd NF, Lockwood GA, Byng JW, Tritchler DL, Yaffe MJ. Mammographic densities and breast cancer risk Cancer Epidemiol Biomarkers Prev 1998; 7:1133-44.

8. Stomper PC, D'Souza DJ, DiNitto PA, Arredondo MA. Analysis of parenchymal density on mammograms in 1353 women 25-79 years old. AJR Am J Roentgenol 1996; 167:1261-5.

9. Van Gils $\mathrm{CH}$. Mammographic density and breast cancer risk. Eur J Obstet Gynecol Reprod Biol 1999; 86:127-8.

10.Ma L, Fishell E, Wright B, Hanna W, Allan S, Boyd NF. Case control study of factors associated with failure to detect breast cancer by mammography. J Natl Cancer Inst 1992; 84:781-5.

11.Chlebowski RT, Hendrix SL, Langer RD, et al. Influence of estrogen plus progestin on breast cancer and mammography in healthy postmenopausal women: the Women's Health Initiative Randomized Trial. JAMA 2003; 289:3243-53.

12.Van Gils CH, Otten JD, Verbeek AL, Hendriks JH, Holland R. Effect of mammographic breast density on breast cancer screening performance: a study in Nijmegen, the Netherlands. J Epidemiol Community Health 1998; 52:267-71. 
13.Santos CC. Efeitos da terapia de reposição hormonal na densidade mamográfica de mulheres menopausadas [tese]. Campinas: Univ. de Campinas; 1997.

14. Organização Mundial de Saúde. Obesity: preventing and managing the global epidemic. Report of a WHO consultation on obesity. Geneva: WHO; 1997.

15.Hosmer DW, Lemeshow S. Applied logistic regression. $1^{\text {st }}$ ed. New York: John Wiley \& Sons; 1989.

16. Bergkvist L, Tabar L, Bergström R, Adami HO. Epidemiologic determinants of the mammographic parenchymal pattern. A population-based study within a mammographic screening program. Am. J Epidemiol 1987, 126:1075-81.

17.Van Gils CH, Otten JD, Verbeek AL, Hendriks JH. Short communication: breast parenchymal patterns and their changes with age. Br J Radiol 1995; 68:1133-5.

18. Grove JS, Goodman MJ, Gilbert FI Jr, Mi MP. Factors associated with mammographic pattern. $\mathrm{Br} \mathrm{J}$ Radiol 1985; 58:21-5.

19.Gram IT, Funkhouser E, Tabar L. Anthropometric indices in relation to mammographic patterns among peri-menopausal women. Int J Cancer 1997;
73:323-6.

20.Brisson J, Morrison AS, Kopans DB, et al. Height and weight, mammographic features of breast tissue, and breast cancer risk. Am J Epidemiol 1984; 119:371-81.

21.Speroff L. The meaning of mammographic breast density in users of postmenopausal hormone therapy. Maturitas 2002; 41:171-5.

22.Gram IT, Funkhouser E, Tabar L. Reproductive and menstrual factors in relation to mammographic parenchymal patterns among perimenopausal women. Br J Cancer 1995; 71:647-50.

23. Colacursi N, Fornaro F, De Franciscis P, Mele D, Palermo M, del Vecchio W. Effects of a short-term suspension of hormone replacement therapy on mammographic density. Fertil Steril 2001; 76:451-5.

24.Christodoulakos GE, Lambrinoudaki IV, Vourtsi AD, Panoulis KP, Kelekis DA, Creatsas GC. Mammographic changes associated with raloxifene and tibolone therapy in postmenopausal women: a prospective study. Menopause 2002; 9:110-6.

Recebido em: 30/10/2003 Aceito com modificações em: 7/1/2004 